\title{
Estratégias para o desenvolvimento de educação em saúde sobre dor no hospital
}

\author{
Strategies for the development of health education on pain in the hospital \\ Estrategias para el desarrollo de educación em salud sobre dolor em el hospital
}

Recebido: 25/02/2021 | Revisado: 03/03/2021 | Aceito: 06/03/2021 | Publicado: 14/03/2021

\author{
Fernanda Westphal Hahn \\ ORCID: https://orcid.org/0000-0002-0951-760X \\ Universidade Federal de Pelotas, Brasil \\ E-mail: fernandahahn6@gmail.com \\ Franciele Roberta Cordeiro \\ ORCID: https://orcid.org/0000-0001-6194-5057 \\ Universidade Federal de Pelotas, Brasil \\ E-mail: franciele.cordeiro@ufpel.edu.br
}

\begin{abstract}
Resumo
A avaliação e a intervenção sobre a dor devem ser atividades prioritárias na atuação profissional no contexto hospitalar, pois ela impacta na qualidade de vida. Por isso, esta revisão integrativa teve por objetivo identificar, na literatura brasileira e internacional, estratégias para o desenvolvimento de educação em saúde sobre dor no hospital. A seleção dos estudos ocorreu, entre fevereiro e março de 2020, nas bases de dados Scientific Electronic Library Online (SCIELO), Literatura Latino-Americana e do Caribe em Ciências da Saúde (LILACS), Scopus, PubMed e Web of Science. Foram identificados 449 artigos e, dentre eles, após a aplicação dos critérios de inclusão e exclusão, 17 compuseram o material empírico de análise. As informações extraídas dos estudos foram organizadas em planilha do programa Microsoft Excel, avaliou-se o nível de evidência das publicações, e a análise ocorreu com base na síntese e proximidade dos tipos de estratégias de educação em saúde. Os resultados abordaram três grupos de estratégias recomendadas para uso com profissionais de saúde, pacientes e cuidadores, a citar: tecnologias digitais, estratégias grupais e psicoeducativas, e cartilhas e materiais impressos. Constatou-se que os estudos apresentaram, em sua maioria, forte recomendação com base no nível de evidência. Vídeos, jogos, cartilhas, psicodrama e plataformas online são estratégias mais atraentes, interativas e acessíveis. Essas estratégias podem ser incorporadas durante a hospitalização, a fim de favorecer a compreensão e o protagonismo de pacientes e familiares no controle da dor, bem como pode ser utilizada com estudantes e profissionais de saúde.
\end{abstract}

Palavras-chave: Revisão; Dor; Educação em saúde; Hospitais.

\begin{abstract}
The evaluation and intervention on pain should be priority activities in the professional performance in the hospital context since it impacts the quality of life. Therefore, this integrative literature review aimed to identify, in the Brazilian and international literature, strategies for the development of health education on pain in the hospital. The selection of the studies took place, between February and March 2020, in the databases Scientific Electronic Library Online (SCIELO), Literatura Latino-Americana e do Caribe em Ciências da Saúde (LILACS), Scopus, Pubmed, and Web of Science. We identified 449 articles and, among them, after applying the inclusion and exclusion criteria, 17 composed the empirical material of analysis. We organized the information extracted from the studies in a spreadsheet of the Microsoft Excel program, the level of evidence of the publications was evaluated, and the analysis was based on the synthesis and proximity of the types of health education strategies. The results addressed three groups of strategies recommended for use with health professionals, patients, and caregivers, including digital technologies, group and psycho-educational strategies, and booklets and printed materials. We found that most of the studies presented a strong recommendation based on the level of evidence. Videos, games, booklets, psychodrama, and online platforms are more attractive, interactive, and accessible strategies. These strategies can be incorporated during hospitalization, in order to favor the understanding and protagonism of patients and their caregivers in pain control, as well as can be used with students and health professionals.
\end{abstract}

Keywords: Review; Pain; Health education; Hospitals.

\section{Resumen}

La evaluación y la intervención sobre el dolor deben ser actividades prioritarias en el desempeño profesional en el contexto hospitalario ya que impacta en la calidad de vida. Por lo tanto, esta revisión de la literatura integradora tuvo como objetivo identificar, en la literatura brasileña e internacional, estrategias para el desarrollo de la educación sanitaria sobre el dolor en el hospital. La selección de los estudios ocurrió, entre febrero y marzo de 2020, en las bases de datos Scientific Electronic Library Online (SCIELO), Literatura Latino-Americana e do Caribe em Ciências da 
Saúde (LILACS), Scopus, Pubmed y Web of Science. Se identificaron 449 artículos y, de entre ellos, tras la aplicación de los criterios de inclusión y exclusión, 17 compusieron el material empírico de análisis. La información extraída de los estudios se organizó en hoja de cálculo del programa Microsoft Excel, se evaluó el nivel de evidencia de las publicaciones, y el análisis se llevó a cabo sobre la base de la síntesis y la proximidad de los tipos de estrategias de educación en salud.Los resultados abordaron tres grupos de estrategias recomendadas para su uso con profesionales de la salud, pacientes y cuidadores: tecnologías digitales, estrategias grupales y psicoeducativas, y cartillas y materiales impresos. Se constató que la mayoría de los estudios presentaban una fuerte recomendación basada en el nivel de evidencia. Videos, juegos, cartillas, psicodrama y plataformas en línea son estrategias más atractivas, interactivas y asequibles. Estas estrategias pueden ser incorporadas durante la hospitalización, con el fin de favorecer la comprensión y el protagonismo de pacientes y familiares en el control del dolor, así como puede ser utilizada con estudiantes y profesionales de la salud.

Palabras clave: Revisión; Dolor; Educación en salud; Hospitales.

\section{Introdução}

A dor é uma experiência sensorial e emocional desagradável que está relacionada a um dano tecidual real ou potencial (Santana, Perissinotti, Oliveira Junior, Correia, Oliveira \& Fonseca, 2020; Iasp, 2017). Classifica-se em dor nociceptiva (podendo ser visceral ou somática) ou neuropática. A dor nociceptiva ocorre quando há presença de traumatismo e inflamação, e a dor neuropática é causada por lesão ou doença que afeta o sistema somatossensorial (Miranda, Seda Júnior \& Pelloso, 2016).

Também pode ser classificada de acordo com o tempo de duração, podendo ser aguda ou crônica. A dor aguda tem início súbito e está relacionada a traumas, infecções e inflamações, e espera-se que desapareça após intervenção farmacológica ou não-farmacológica (Iasp, 2017). A dor crônica permite adaptação do organismo à determinada situação (Alves Neto et al, 2009). Quanto à cronicidade, não há consenso, visto que documento brasileiro considera como critério para sua definição a duração superior a trinta dias (Brasil, 2012) e documentos internacionais consideram um período maior que três meses, e a sua exacerbação (Nanda-I, 2018; Moisset et al, 2020).

Nos Estados Unidos da América, estima-se que um a cada cinco adultos sinta dor crônica (Kuehn, 2018). No Brasil, um estudo (Pedroso et al., 2017) realizado com pacientes oncológicos que buscou conhecer a percepção da dor em oncologia na ótica do paciente e dos profissionais de enfermagem, identificou que os pacientes expressam sua dor envolvendo não somente aspectos físicos, mas também emocionais, sociais e espirituais. No mesmo estudo, enfermeiros demonstraram preocupação em compreender e aliviar a dor desses pacientes, recorrendo não somente às medidas farmacológicas, mas ao vínculo e ao apoio (Pedroso et al., 2017).

Em 2018, relatório publicado pela Comissão Lancet sobre as diferenças no acesso aos cuidados paliativos e às medidas de alívio do sofrimento pela dor identificou que países com elevado desenvolvimento econômico apresentam intervenções mais eficazes quando comparados aos países de baixo ou médio desenvolvimento econômico. Nesses últimos, de maneira geral, a população tem pouco ou nenhum acesso ao alívio da dor ou cuidados paliativos (Knaul et al, 2018).

Dessa forma, avaliar e intervir sobre a dor deve ser uma das atividades prioritárias dos profissionais de saúde, no ambiente domiciliar, ambulatorial e hospitalar, pois esse sintoma tem sido um dos principais motivos de busca dos usuários por atendimento nos serviços de saúde, notadamente, nos serviços de urgência e emergência (Dick \& Lohmann, 2020).

Especificamente sobre o hospital, embora a dor tenha sido reconhecida há mais de uma década como o quinto sinal vital, equipes de saúde ainda apresentam resistência ou dificuldade para avaliar, tratar e educar pacientes e cuidadores, informais e formais, sobre esse sintoma. Uma limitação identificada desde a formação (Ferrari, Daher, Antunes, Amim, Jesus \& Geraldo, 2019).

Frente ao exposto, se faz necessário o desenvolvimento de ações de educação junto aos profissionais, mas também ações de educação em saúde direcionadas aos pacientes e cuidadores, no sentido de ampliar a participação desses no processo 
de avaliação, acompanhamento e controle da dor. Por isso, este estudo teve como objetivo identificar, na literatura brasileira e internacional, estratégias para o desenvolvimento de educação em saúde sobre dor no hospital.

\section{Metodologia}

Trata-se de um estudo de abordagem qualitativa, do tipo revisão integrativa de literatura, que seguiu as etapas propostas de Mendes, Silveira e Galvão (2019), a citar: definição da questão da revisão, busca e seleção de estudos primários, extração de dados, avaliação dos estudos, síntese dos resultados e apresentação.

Na primeira etapa, delimitou-se como questões de pesquisa: quais estratégias de educação em saúde sobre dor são identificadas na literatura internacional? Qual o nível de evidência das publicações que apresentam ações de educação em saúde sobre dor? Essa questão foi construída com base na estratégia PICO, ou seja, (População - P: paciente, cuidador ou profissional de saúde; Intervenção - I: ações de educação em saúde sobre dor; Comparação - C: não se aplica; "Outcomes"/desfecho - O: melhora da avaliação ou sensação de dor).

Na segunda etapa, busca e seleção dos estudos primários, foram associados os descritores em ciências da saúde (DECS) "dor" e "educação em saúde" com o operador booleano <AND $>$ na base de dados Scientific Electronic Library Online (SCIELO), descritores "dor", "cuidados paliativos" e "adulto" com o operador booleano <AND $>$ na base de dados Literatura Latino-Americana e do Caribe em Ciências da Saúde (LILACS), e Medical Subject Headings (MeSH) "educational technology", "pain", "health education" e "child" com os operadores booleanos $<$ AND $>$ e $<$ NOT $>$ nas bases de dados Scopus, PubMed e Web of Science.

Definiu-se como critérios de inclusão: artigos originais, artigos de revisão, relatos de experiências, publicados em português, inglês e espanhol e, que abordaram especificamente estratégias de orientação e educação em relação à dor. Foram excluídas monografias, editoriais, e artigos de reflexão. Não foi estabelecida delimitação temporal, de forma a contemplar o maior número de estudos possíveis.

Assim, identificou-se 449 artigos dos quais foram lidos, inicialmente, o título e resumo. Após essa etapa, 33 artigos foram selecionados para leitura na íntegra no período de $1^{\circ}$ de fevereiro a 15 de março de 2020. Após empregar os critérios de inclusão e exclusão, 17 artigos compuseram o material empírico de análise. A Figura 1 apresenta um diagrama com o processo de identificação e seleção dos estudos.

Figura 1. Diagrama do processo de identificação e seleção dos estudos.

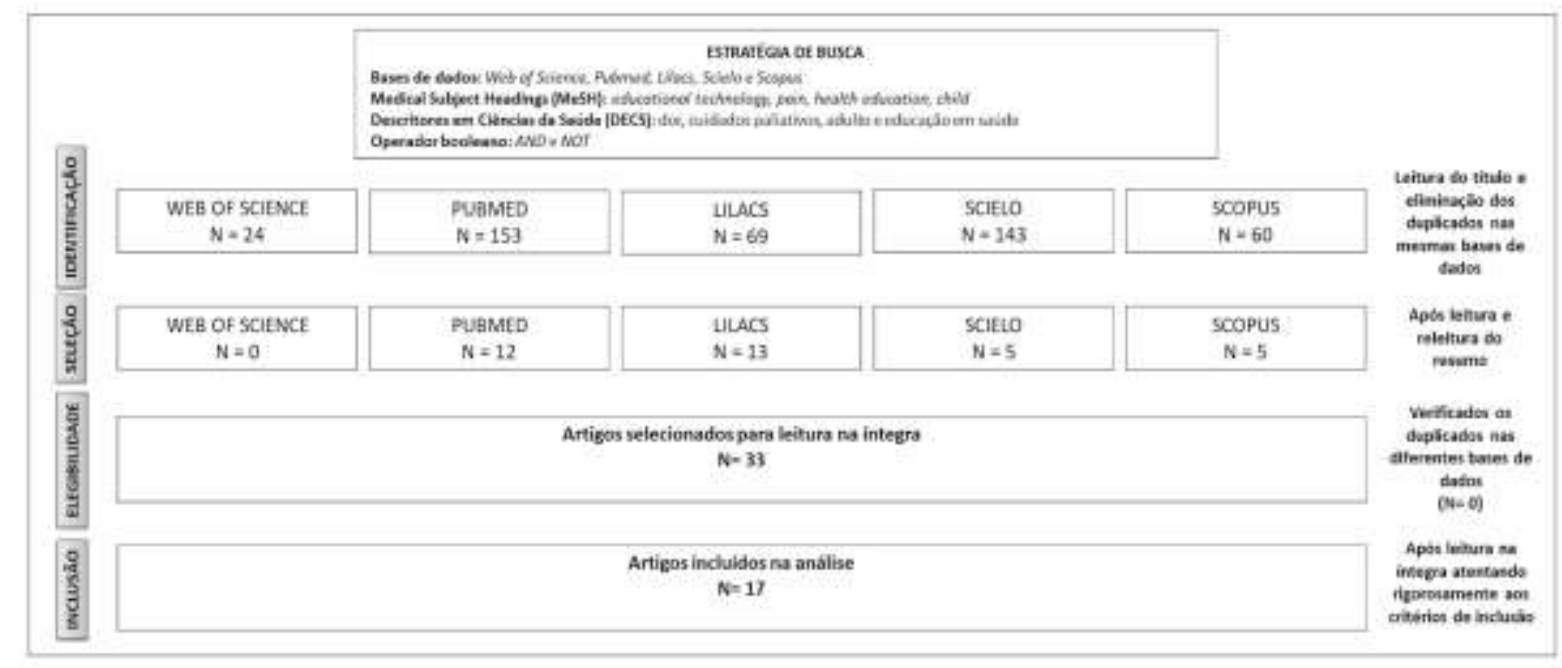

Fonte: Dados da pesquisa (2021). 
Na terceira etapa, extraiu-se os seguintes dados dos estudos: título, revista, autor, ano, país, objetivo do estudo, referencial teórico, desenho metodológico, tipo de amostragem, intervenções, participantes, técnica de coleta, organização e análise de dados, aspectos éticos, fator de impacto das revistas, nível de evidência dos estudos, ações de educação em saúde realizadas, conclusão e limitações. O nível de evidência foi avaliado conforme proposta de Melnyk e Fineout-Overholt (2019). Destaca-se que para a quarta etapa, avaliação dos estudos, optou-se pela avaliação do nível de evidência, uma das possibilidades apontadas por Mendes, Silveira e Galvão (2019), no percurso metodológico da revisão integrativa.

$\mathrm{Na}$ quinta e sexta etapas, os dados foram organizados em planilhas no programa Microsoft Excel, conforme exemplificado na Figura 2. Aqueles relativos à caracterização dos estudos foram analisados por frequência absoluta. Os referentes às estratégias utilizadas para o controle da dor foram reunidos conforme a similitude do tipo de ação e apresentados de forma narrativa.

Figura 2. Organização dos dados.

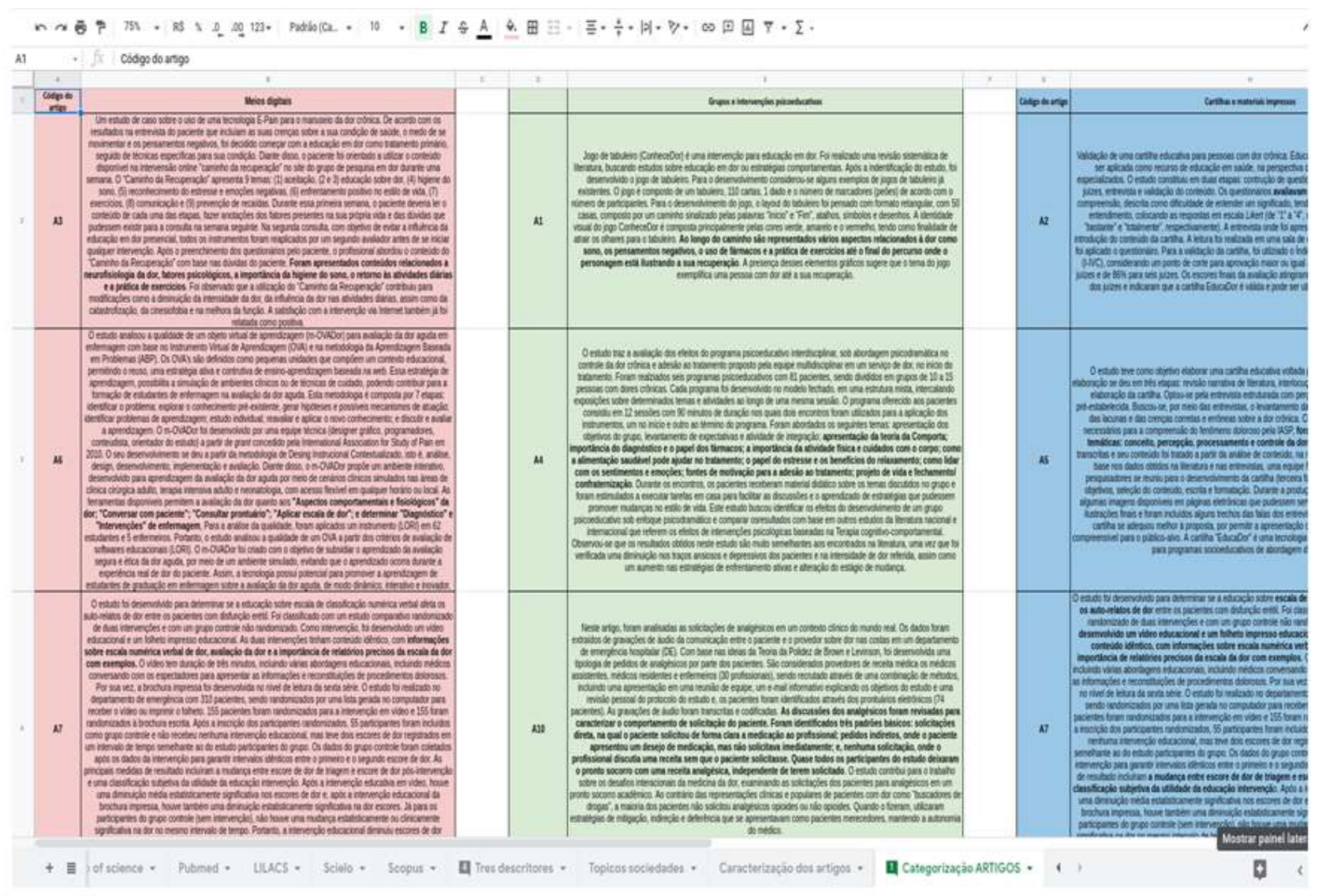

Fonte: Dados da pesquisa (2021).

\section{Resultados}

Após a leitura dos artigos na íntegra e extração dos resultados, 17 artigos compuseram o material de análise. Tais artigos são apresentados no Quadro 1. 
Quadro 1. Material empírico analisados.

\begin{tabular}{|c|c|c|c|c|}
\hline Autores & Título & Ano & Periódico & $\begin{array}{l}\text { Nível de } \\
\text { evidência }\end{array}$ \\
\hline Chu, T.L. et al. & $\begin{array}{l}\text { Multimedia-assisted instruction on pain } \\
\text { assessment learning of new nurses: a } \\
\text { quasi-experimental study }\end{array}$ & 2019 & $\begin{array}{l}\text { BMC Medical } \\
\text { Education }\end{array}$ & III \\
\hline Valentim, J. C. et al. & $\begin{array}{l}\text { ConheceDOR: the development of board } \\
\text { game for modern pain education for } \\
\text { patients with musculoskeletal pain }\end{array}$ & 2019 & $\begin{array}{l}\text { Brazilian Journal of } \\
\quad \text { Pain (BrJP) }\end{array}$ & II \\
\hline Vieira, A.S.M. et al. & $\begin{array}{l}\text { Validation of an educational booklet for } \\
\text { people with chronic pain: EducaDor }\end{array}$ & 2019 & $\begin{array}{l}\text { Brazilian Journal of } \\
\quad \text { Pain (BrJP) }\end{array}$ & III \\
\hline Alvarez, A.G. et al. & $\begin{array}{l}\text { Quality analysis of the virtual learning } \\
\text { object for nursing pain assessment }\end{array}$ & 2018 & $\begin{array}{l}\text { Revista Cubana de } \\
\text { Enfermería }\end{array}$ & III \\
\hline Lima, L.; Reis, F. & $\begin{array}{l}\text { The use of na E-pain technology in the } \\
\text { management of chronic pain. Case report }\end{array}$ & 2018 & $\begin{array}{l}\text { Brazilian Journal of } \\
\quad \text { Pain (BrJP) }\end{array}$ & VI \\
\hline Loduca, A. et al. & $\begin{array}{c}\text { How interdisciplinary psychoeducational } \\
\text { programs with a psychodrama approach } \\
\text { can help the chronic pain treatment } \\
\text { compliance }\end{array}$ & 2018 & $\begin{array}{l}\text { Brazilian Journal of } \\
\quad \text { Pain (BrJP) }\end{array}$ & III \\
\hline Sullivan, M. et al. & $\begin{array}{l}\text { A Controlled Pilot Trial of PainTracker } \\
\text { Self-Manager, a Web-Based Platform } \\
\text { Combined With Patient Coaching, to } \\
\text { Support Patients' Self-Management of } \\
\text { Chronic Pain }\end{array}$ & 2018 & The Journal of Pain & III \\
\hline Mendez, S. P. et al. & $\begin{array}{l}\text { Elaboration of a booklet for individuals } \\
\text { with chronic pain }\end{array}$ & 2017 & Revista Dor & VI \\
\hline $\begin{array}{l}\text { Buchbinder, M.; } \\
\text { Wilbur, R.; McLean, S.; } \\
\text { Sleath, B. }\end{array}$ & $\begin{array}{c}\text { "Is there any way I can get something for } \\
\text { my pain?" Patient strategies for requesting } \\
\text { analgesics }\end{array}$ & 2015 & $\begin{array}{l}\text { Patient Education } \\
\text { and Counseling }\end{array}$ & VI \\
\hline $\begin{array}{l}\text { Capewell, C.; Gregory, } \\
\text { W.; Closs, S.; Bennett, } \\
\text { M. }\end{array}$ & $\begin{array}{l}\text { Brief DVD-based educational intervention } \\
\text { for patients with cancer pain: feasibility } \\
\text { study }\end{array}$ & 2010 & Palliative Medicine & II \\
\hline Street, R.L Jr. et al. & $\begin{array}{l}\text { Improving physician-patient } \\
\text { communication about cancer pain with a } \\
\text { tailored education-coaching intervention }\end{array}$ & 2010 & $\begin{array}{l}\text { Patient Education } \\
\text { and Counseling }\end{array}$ & II \\
\hline Syrjala, K.L. et al. & $\begin{array}{l}\text { Patient training in cancer pain management } \\
\text { using integrated print and video materials: } \\
\text { a multisite randomized controlled trial }\end{array}$ & 2008 & Revista Pain & II \\
\hline
\end{tabular}




\begin{tabular}{|c|c|c|c|c|}
\hline Marco, C. A. et al. & $\begin{array}{c}\text { The Verbal Numeric Pain Scale: Effects of } \\
\text { Patient Education on Self-reports of Pain }\end{array}$ & 2006 & $\begin{array}{c}\text { Society for } \\
\text { Academic } \\
\text { Emergency } \\
\text { Medicine }\end{array}$ & II \\
\hline Yates, P. et al. & $\begin{array}{c}\text { A randomized controlled trial of a nurse } \\
\text { administered educational intervention for } \\
\text { improving cancer pain management in } \\
\text { ambulatory settings }\end{array}$ & 2004 & $\begin{array}{c}\text { Patient Education } \\
\text { and Counseling }\end{array}$ & II \\
\hline West, C. M. et al. & $\begin{array}{c}\text { The PRO-SELF(c): Pain Control Program- } \\
\text {-an effective approach for cancer pain } \\
\text { management }\end{array}$ & 2003 & $\begin{array}{c}\text { Oncology Nursing } \\
\text { Forum }\end{array}$ & VI \\
\hline Edwards, H.E. et al. & $\begin{array}{c}\text { Improving pain management by nurses: A } \\
\text { pilot Peer Intervention Program }\end{array}$ & 2001 & $\begin{array}{c}\text { Nursing and Health } \\
\text { Sciences }\end{array}$ & III \\
\hline Erkes, E.B; Parker, & $\begin{array}{c}\text { An examination of critical care nurses' } \\
\text { knowledge and attitudes regarding pain } \\
\text { Ranagement in hospitalized patients }\end{array}$ & 2001 & $\begin{array}{c}\text { Revista Pain } \\
\text { Management } \\
\text { Nursing }\end{array}$ & III \\
\hline
\end{tabular}

Fonte: Dados da pesquisa (2021).

Em relação à caracterização, 2018 foi o ano com maior número de publicações, com quatro estudos (Lima \& Reis, 2018; Loduca et al., 2018; Alvarez, et al., 2018; Sullivan et al., 2018), seguido do ano de 2019 com três estudos publicados (Valentim et al., 2019; Vieira et al., 2019; Chu et al., 2019). Estados Unidos da América (Marco et al., 2006; Sullivan et al., 2018; Buchbinder, Wilbur, Mclean \& Leath, 2015; Street et al., 2010; Syrjala et al., 2008; West et al., 2003; Erkes, Parker; Carr \& Mayo, 2001) e Brasil (Valentim et al., 2019; Vieira et al., 2019; Lima \& Reis, 2018; Loduca et al., 2018; Mendez et al., 2017; Alvarez et al., 2018) foram os dois países com maior número mais publicações sobre o tema, tendo sete e seis artigos publicados respectivamente.

Quanto ao delineamento, 12 estudos utilizaram a abordagem quantitativa (Vieira et al., 2019; Loduca et al., 2018; Alvarez et al., 2018; Marco et al., 2006; Chu et al., 2019; Sullivan et al., 2018; Capewell; Gregory, Closs \& Bennett, 2010; Street et al., 2010; Syrjala et al., 2008; Yates et al., 2004; Edwards et al., 2001; Erkes, Parkerg, Carr \& Mayo, 2001) e cinco utilizaram a abordagem qualitativa (Valentim et al., 2019; Lima \& Reis, 2018; Mendez et al., 2017; Buchbinder; Wilbur; Mclean \& Sleath, 2015; West et al., 2003).

Sobre o tipo de amostragem, quando mencionada, 12 estudos utilizaram a forma intencional (Valentim et al., 2019; Vieira et al., 2019; Lima \& Reis, 2018; Loduca et al., 2018; Mendez et al., 2017; Alvarez et al., 2018; Chu et al., 2019; Sullivan et al., 2018; Buchbinder; Wilbur; Mclean \& Sleath, 2015; West et al., 2003; Edwards et al., 2001; Erkes, Parkerg, Carr \& Mayo, 2001) e cinco a randomizada (Marco et al., 2006; Capewell, Gregory, Closs \& Bennett, 2010; Street et al., 2010; Syrjala et al., 2008; Yates et al., 2004).

A respeito dos participantes, dois estudos apresentaram como participantes enfermeiros, médicos e pacientes (Buchbinder, Wilbur, Mclean, Sleath, 2015; Syrjala et al., 2008), oito somente pacientes (Vieira et al., 2019; Lima \& Reis, 2018; Loduca et al., 2018; Mendez et al., 2017; Marco et al., 2006; Sullivan et al., 2018; Yates et al., 2004; West et al., 2003), um com pacientes e familiares (Capewell, Gregory, Closs \& Bennett, 2010), um com pacientes e médicos (Street et al., 2010), 
um com enfermeiros e estudantes (Alvarez et al., 2018), e três somente com enfermeiros (Chu et al., 2019; Edwards et al., 2001; Yates et al., 2004).

Quanto à análise de dados, nove estudos utilizaram estatística descritiva (Vieira et al., 2019; Loduca et al., 2018; Alvarez et al., 2018; Marco et al., 2006; Sullivan et al., 2018; Capewell, Gregory, Closs \& Bennett, 2010; Syrjala et al., 2008; Yates et al., 2004; Erkes, Parkerg, Carr \& Mayo, 2001), seis empregaram análise do conteúdo (Valentim et al., 2019; Mendez et al., 2017; Chu et al., 2019; Buchbinder, Wilbur, Mclean, Sleath, 2015; Street et al., 2010; West et al., 2003) e dois recorreram à análise de conteúdo em combinação com estatística descritiva (Lima \& Reis, 2018; Edwards et al., 2001).

Em relação ao fator de impacto dos periódicos onde foram publicados os estudos, "The Journal of Pain" (5.424), "Palliative Medicine" (4.956), "Society for Academic Emergency Medicine" (2.963) e "Patient Education and Counseling" (2.821), foram aquelas com maior impacto. Por fim, quatro estudos nortearam suas análises a partir de referenciais teóricos, a citar: Teoria da polidez de Levinson (Buchbinder, Wilbur, Mclean, Sleath, 2015), Teoria social cognitiva (Street et al., 2010), Teoria do Comportamento Planejado (Edwards et al., 2001) e Modelo PRECEDE de Green de comportamento em saúde (Yates et al., 2004).

Após a interpretação dos dados, buscando identificar as principais ações de educação em saúde sobre dor no ambiente hospitalar, foram construídas três unidades analíticas: "Tecnologias digitais", "Estratégias grupais e intervenções psicoeducativas" e "Cartilhas e materiais impressos".

\section{Tecnologias digitais}

Nove estudos trouxeram como estratégia de educação sobre dor as tecnologias digitais (Lima \& Reis, 2018; Alvarez et al., 2018; Marco et al., 2006; Chu et al., 2019; Sullivan et al., 2018; Capewell, Gregory, Closs \& Bennett, 2010; Syrjala et al., 2008; West et al., 2003; Erkes, Parker, Carr \& Mayo, 2001).

Os estudos nessa perspectiva abordaram, em sua maioria, informações sobre mecanismos que provocam a dor, bem como a importância de estimular pacientes a se comunicarem com a equipe de profissionais da saúde e de participarem na avaliação e controle da dor. Apresentam orientações sobre uso de medicamentos como mitos e medos sobre o uso de fármacos opioides, esclarecimentos sobre titulação dessa classe de analgésicos e seus efeitos colaterais. Além disso, alguns estudos trouxeram a importância de capacitar profissionais da saúde em relação à avaliação e ao manejo da dor.

O estudo de Lima e Reis (2018) desenvolveu uma intervenção intitulada "caminho da recuperação", disponível de maneira online no site do grupo de pesquisa. A intervenção constituiu-se de nove temas, sendo eles: aceitação, educação sobre dor, higiene do sono, reconhecimento do estresse e emoções negativas, enfrentamento positivo no estilo de vida, exercícios, comunicação e prevenção de recaídas. O paciente pode ler os conteúdos ofertados e fazer anotações dos fatores que se encaixavam em sua condição de saúde, de modo a abordá-los nas consultas seguintes. Após a intervenção, foi observada diminuição da intensidade da dor, da interferência desse sintoma nas atividades diárias, assim como a cinesiofobia (medo de sentir dor) do paciente (Lima \& Reis, 2018).

Sullivan et al. (2018) relataram o desenvolvimento e o teste de um instrumento na web de autogerenciamento (PainTracke). Esse instrumento fornece aos profissionais e pacientes um entendimento maior sobre a experiência do paciente com dor crônica e a resposta ao tratamento ao longo tempo, avaliando o sono, o estado emocional e funcional. O PainTracke foi estendido a uma ferramenta de suporte de autogestão usando os conceitos da Terapia de Aceitação e Compromisso (TCA), que apresenta três componentes principais: 1) aumentar a conscientização do paciente sobre as experiências em andamento, 2) aumentar a abertura às experiências e 3) promover níveis crescentes de participação nas atividades que eles valorizam. Para a realização da intervenção, os pacientes foram direcionados a uma plataforma online onde foram abordados tópicos como: utilização da plataforma, o que é dor, sistema de navegação vitalícia (desenvolvimento de um plano de ação para melhorar uma 
área de vida selecionada), get ritmo (ritmo, respiração consciente e ritmos do sono), e life goes on (agendar atividades baseadas em valor e gerenciar a dor a longo prazo).

Além disso, os pacientes tiveram treinamentos de maneira presencial, por telefone e por e-mail com enfermeiros ou assistente social. Este estudo forneceu evidências de que instrumentos oriundos do TCA e baseados em ferramentas de educação e avaliação para dor crônica, associados a treinamentos com profissionais é eficaz para melhorar a auto eficácia da dor, envolvimento da intensidade e interferência da dor, e satisfação do tratamento, desenvolvendo o empoderamento dos pacientes com dor crônica (Sullivan et al, 2018).

Alvarez et al. (2018) analisaram a qualidade de um objeto virtual de aprendizagem (m-OVADor) para avaliação da dor aguda em enfermagem com base no Instrumento Virtual de Aprendizagem (OVA) e na metodologia da Aprendizagem Baseada em Problemas (ABP). Essa estratégia de aprendizagem possibilita a simulação de ambientes clínicos ou de técnicas de cuidado, podendo contribuir para a formação de estudantes de enfermagem na avaliação da dor aguda. A tecnologia promove a aprendizagem de estudantes de graduação em enfermagem sobre a avaliação da dor aguda, de modo dinâmico, interativo e inovador.

Marco et al. (2006) investigaram se a realização de educação sobre escala de classificação numérica verbal afetaria ou não os autorrelatos de dor. Para isso, elaboraram um vídeo e um folheto impresso educacional. O conteúdo do vídeo versava em torno de informações sobre escala numérica verbal de dor, avaliação da dor e a importância de relatórios precisos da escala da dor com exemplos. O vídeo apresentou diversas abordagens educacionais como médicos dialogando com os espectadores, de maneira que apresentassem as informações e reconstituições de procedimentos dolorosos. Houve diminuição nos escores de dor nos pacientes que receberam a intervenção.

Chu et al. (2019) utilizaram multimídia e instruções em sala de aula para ensinar conhecimentos e habilidades sobre dor aos enfermeiros recém formados, com o intuito de aumentar a capacidade de avaliação e tratamento da dor através de simulações de cenários. O vídeo (DVD) sobre avaliação da dor foi baseado nos cinco componentes do modelo instrucional ADDIE - Análise, design, desenvolvimento, implementação e avaliação. Na análise, foram realizados resumos dos registros de treinamento de avaliação da dor do ano anterior relacionados à capacidade dos novos enfermeiros de lidar com a avaliação da dor. Já no design, foram abordados conteúdos de instrução como: importância da avaliação e tratamento da dor, compreensão e avaliação da dor, causas prováveis da dor, e métodos de tratamento.

O estudo ofertou instrução em sala de aula sobre cuidados com a dor, além de apresentações de profissionais que utilizaram PowerPoint e DVD's instrucionais como material didático de intervenção. Os enfermeiros recém formados apresentaram uma impressão positiva do conteúdo do material, fazendo com que os autores percebessem que a abordagem utilizada facilitava a concentração e satisfação com o aprendizado (Chu et al, 2019).

Capewell, Gregory, Closs \& Bennett (2010) desenvolveram um DVD educacional e um folheto para pacientes com dor de câncer avançado e seus cuidadores com o intuito de testar a viabilidade e avaliar essa intervenção. O DVD continha entrevistas com uma equipe multidisciplinar de cuidados paliativos e abordava aspectos da dor no câncer e uso de opioides. Também foi abordada a importância da avaliação da dor e a boa comunicação. O estudo observou melhorias significativas sobre conhecimentos e atitudes dos participantes, mas pequenas melhorias não significativas para a intensidade da dor.

Syrjala et al. (2008) testaram um método de treinamento através de vídeo e manual com conteúdos padronizados. O vídeo e manual foram direcionados para pacientes que apresentavam dor relacionada ao câncer, devendo estar saudável o suficiente para aprender e aplicar novas informações. O conteúdo dos materiais foram determinados através de um painel de especialistas clínicos de câncer na comunidade. Os materiais foram projetados para uso diário na prática ambulatorial por enfermeiros e médicos. O vídeo forneceu informações através de pacientes com câncer e com dor falando sobre suas experiências com o tratamento, abordando conteúdos sobre alívio da dor, efeitos colaterais dos medicamentos e comunicação 
com médicos. O treinamento para dor incluiu assistir o vídeo e revisar o conteúdo no manual com a enfermeira, observando as preocupações individuais de cada paciente. Os autores detectaram que o treinamento da dor com pacientes reduziu significativamente a dor usual e as barreiras ao alívio da dor (Syrjala et al., 2008).

West et al. (2003) descreveram os componentes do PRO-SELF: Pain Control Program, para uso dos enfermeiros que prestam assistência a pacientes com câncer. O PRO-SELF (Programa de controle da dor) foi desenvolvido para fornecer aos pacientes e cuidadores informações, desenvolvimento de habilidades e suporte interativo de enfermagem destinado a ensinar os pacientes a prevenir e controlar os efeitos da sua doença e tratamento. Antes de receber a intervenção, os pacientes foram orientados a registrar seu nível de dor todos os dias antes de dormir por um período de sete dias. Feito isso, destacaram a menor, a média e a pior dor do dia usando uma escala de classificação numérica. Além disso, os participantes completaram um questionário sobre seus conhecimentos em relação a dor do câncer, seu manejo, efeitos colaterais dos analgésicos e conceitos de dependência e tolerância e, receberam um livro de controle da dor (PRO-SELF: Pain Control Book). Na última visita domiciliar, pacientes e cuidados completaram novamente o questionário como forma de avaliar a eficácia do programa.

A capacitação dos pacientes envolveu: desenvolver habilidades em avaliar sua dor e sua resposta aos analgésicos; desenvolver gestão analgésica que melhor atendesse as necessidades de tratamento e estilo de vida; planejar estratégias para prevenção e tratamento de efeitos colaterais dos analgésicos; e conversar com os profissionais de saúde que os acompanhavam caso sintam que a receita analgésica não está adequada. Após aplicar o programa de controle da dor, identificou-se que a ferramenta é eficaz, podendo ser utilizada para ajudar pacientes com câncer e seus cuidadores a terem mais conhecimento e a desenvolver as habilidades necessárias para gerenciar a dor (West et al., 2003).

Por fim, Erkes, Parker, Carr E Mayo (2001) avaliaram a eficácia de uma intervenção educacional focada no tratamento da dor, com o objetivo de examinar os níveis de conhecimento e atitude dos enfermeiros intensivistas frente a esse assunto. Essa intervenção tratava-se de uma fita de vídeo sobre o manejo da dor, bem como um módulo de autoaprendizagem sendo realizado um teste antes e depois do programa educacional. O vídeo abordou as melhores práticas atuais no gerenciamento da dor em pacientes internados, sendo narrada por um médico especialista em oncologia. Após a intervenção, as maiorias dos enfermeiros acertaram mais de $75 \%$ sobre conhecimento e pesquisa de atitude frente ao tema mencionado, melhorando as suas pontuações após a intervenção educacional.

\section{Estratégias grupais e psicoeducativas}

Seis estudos trouxeram como ferramentas de educação sobre dor intervenções psicoeducativas (Valentim et al., 2019; Loduca et al., 2018; Buchbinder, Wilbur, Mclean \& Sleath, 2015; Street et al., 2010; Yates et al., 2004; Edwards et al., 2001).

Os estudos nessa perspectiva abordaram o conhecimento sobre mecanismos que provocam a dor, a importância de estimular pacientes a se comunicar com a equipe, bem como em participar na avaliação e controle da dor, incluindo diálogo para a solicitação de medicamento. Além disso, apresentam orientações sobre o uso de medicamentos e sobre medidas não farmacológicas para a melhora da dor e adesão ao tratamento.

Valentim et al. (2019) desenvolveram um jogo de tabuleiro, intitulado ConheceDor. O jogo tinha um tabuleiro com cinquenta casas, composto de um caminho sinalizado pelas palavras "início" e "fim", atalhos, símbolos e desenhos. Além de cartas, dado e número de marcadores (peões). Essa intervenção abordou aspectos relacionados à dor, como sono, pensamentos negativos, uso de medicamentos e a prática de exercícios. A presença de elementos gráficos no jogo exemplificava uma pessoa com dor até a sua recuperação, contribuindo para o aprendizado dos conceitos e estratégias comportamentais da dor.

Loduca et al. (2018) avaliaram os efeitos de programa psicoeducativo interdisciplinar com abordagem psicodramática no controle da dor crônica e adesão ao tratamento proposto pela equipe multidisciplinar em um serviço de dor, no começo do tratamento. Para isso, foram realizados seis programas psicoeducativos com pacientes acometidos por dor crônica. Cada 
programa foi desenvolvido no modelo fechado, com uma estrutura que intercalava relatos sobre determinados temas e atividades durante a mesma sessão, sendo composta por doze sessões.

A intervenção abordou os seguintes temas: apresentação da Teoria da Comporta; importância do diagnóstico e o papel dos medicamentos; importância da atividade física e cuidados com o corpo; como a alimentação saudável pode ajudar no tratamento; o papel do estresse e os benefícios do relaxamento; maneiras de lidar com os sentimentos e emoções; fontes de motivação para a adesão ao tratamento; projeto de vida e confraternização. Os participantes receberam o material didático sobre os conteúdos abordados e foram incentivados a realizar tarefas em casa a fim de contribuir no aprendizado de estratégias que auxiliem na mudança do estilo de vida (Loduca et al, 2018). Verificou-se diminuição nos traços ansiosos e depressivos dos pacientes e na intensidade da dor referida por eles, assim como um aumento nas estratégias de enfrentamento ativas e a alteração do estágio de mudança (Loduca et al., 2018).

Buchbinder, Wilbur, Mclean E Sleath (2015) analisaram as solicitações de analgésicos em um contexto clínico de um departamento de emergência hospitalar, através de dados extraídos de gravações de áudio da comunicação entre o paciente e o profissional da saúde sobre suas dores. Diante disso, desenvolveram uma tipologia de pedidos de analgésicos por parte dos pacientes com base nas ideias da Teoria da Polidez. Foram identificados três padrões de solicitação: solicitações diretas, na qual o paciente solicitou de forma clara o medicamento ao profissional; solicitações indiretas, onde o paciente apresentou um desejo de medicamento, mas não solicitava imediatamente; e, nenhuma solicitação, na qual o profissional discutia uma receita sem que o paciente solicitasse. Foi observado que todos os participantes do estudo saíram do pronto socorro com uma prescrição analgésica, independente de terem ou não solicitado.

Street et al. (2010) realizaram estudo para verificar a eficácia de uma intervenção de educação e treinamento (TEC) sobre o gerenciamento da dor e maneiras de pacientes conversarem de forma mais eficaz sobre o controle da dor com seus médicos. Essa intervenção foi baseada em princípios da teoria social cognitiva e em modelos de competência em comunicação. Foi realizada uma intervenção educacional e de treinamento sobre medida (TEC) com cuidados usuais educacionalmente aprimorados (EUC). Participaram deste estudo médicos e pacientes que agendaram consulta com os médicos participantes. Ofertou-se aos pacientes uma cópia de um guia sobre controle da dor e, assim, buscou-se avaliar os conhecimentos, atitudes e escolhas atuais em relação ao tratamento da dor; reparar equívocos sobre o controle da dor do câncer; instruir conceitos relevantes relacionados ao controle da dor e a comunicação ativa (ex: importância da realização de perguntas, manifestar angústias e opiniões, entre outras); planejamento (reconhecimento de objetivos e estratégias); e incentivar os pacientes a se manifestarem sobre a dor. Os dois grupos manifestaram entendimentos errôneos acerca da dor, embora o aprendizado tenha facilitado o manejo do sintoma (Street et al, 2010).

Yates et al. (2004) avaliaram a eficácia de uma intervenção educacional a fim de superar obstáculos em relação à atitude e à capacidade dos pacientes de prevenir e gerenciar com mais efetividade a sua dor. Para isso, o estudo foi baseado no modelo PRECEDE de comportamento em saúde, que identifica três categorias de fatores que podem influenciar um comportamento na saúde, como o uso de estratégias para diminuir a dor. A intervenção propõe melhorar o conhecimento e as atitudes dos pacientes em relação à dor do câncer, aumentar a capacidade dos pacientes de se comunicar com os profissionais de saúde sobre o controle da dor e, reduzir a relutância dos pacientes em tomar medicamentos. Os resultados apontaram que a intervenção foi eficaz na redução de preocupações dos pacientes acometidos por dor oncológica e seu tratamento e na disposição dos pacientes, assim como uma melhor comunicação com os profissionais de saúde.

Por fim, Edwards et al. (2001), utilizaram o Programa de Intervenção entre Pares (PIP) visando modificar crenças e atitudes dos enfermeiros sobre narcóticos, identificando as crenças atuais, melhorando seu conhecimento, desmistificando os mitos em torno de narcóticos e discussão em grupo. Os participantes foram divididos em três grupos: 1) intervenção ao paciente (intervenção educacional voltada para o paciente); 2) intervenção enfermeiro-paciente (realizada a implementação de 
intervenção educacional para enfermeiros PIP); e 3) grupo controle (nenhuma intervenção). Os participantes demonstraram confiança na capacidade de realizar atividades relacionadas à administração de analgesia narcótica em pacientes com dor. Os dados do estudo mostram que o PIP foi capaz de melhorar crenças e percepções dos enfermeiros sobre o controle e auto eficácia dos narcóticos, sendo bem recebido pelos participantes.

\section{Cartilhas e materiais impressos}

Seis estudos trouxeram como ferramentas de educação sobre dor cartilhas e materiais impressos (Mendez et al., 2017, Vieira et al., 2019, Marco et al., 2006, Capewell, Gregory, Closs \& Bennett, 2010, Syrjala et al., 2008; West et al., 2003).

Os estudos nessa perspectiva abordaram conhecimentos sobre mecanismos que provocam a dor, importância de estimular o paciente a se comunicar com a equipe e participação na avaliação e controle da dor, orientações sobre o uso de medicações, e esclarecimento sobre titulação de opioides e efeitos colaterais. Além disso, orientações sobre medidas não farmacológicas para a melhora da dor, benefícios de relaxamento, maneiras de lidar com sentimentos e emoções, fontes de motivação para adesão ao tratamento e readaptação do estilo de vida.

Mendez et al. (2017) elaboraram cartilha educativa voltada para pessoas acometidas por dor crônica. A cartilha chamado EducaDor apresentou as seguintes seções: o que é dor? dor aguda: dor útil; dor crônica: a dor persistente; viver com dor; falsas ideias sobre dor crônica, não acredite nelas; e estratégias para lidar com a dor. A cartilha fornece, ainda, conteúdos sobre neurofisiologia e aspectos psicológicos e comportamentais relacionados à dor crônica. Vieira et al. (2019) realizaram a validação da cartilha EducaDor, e após os autores analisaram os resultados da avaliação dos especialistas e observaram que atingiram o nível de excelência e que eles indicaram a cartilha EducaDor como uma intervenção válida e que poderia ser utilizada como recurso terapêutico.

Marco et al. (2006) investigaram se a realização de educação sobre escala de classificação numérica verbal afetaria ou não os autorrelatos de dor. Para isso, elaboraram um vídeo e um folheto impresso educacional que continham conteúdos idênticos com informações sobre escala numérica verbal de dor, avaliação da dor e a importância de relatórios precisos da escala da dor com exemplos. $O$ folheto impresso foi elaborado no nível de sexta série. O estudo realizou intervenções com dois grupos: um grupo recebeu o vídeo e outro a brochura impressa, além de um grupo controle que não receberia nenhuma intervenção. Após a intervenção educacional do folheto impresso, houve diminuição nos escores de dor. Para o grupo controle, não houve mudança na dor.

Capewell, Gregory, Closs e Bennett (2010) desenvolveram um DVD educacional e um folheto para pacientes com câncer avançado e seus cuidadores com o intuito de testar a viabilidade e avaliar essa intervenção. Os participantes foram recrutados em serviços de cuidados paliativos. O folheto apresentava informações sobre aspectos da dor no câncer e uso de opioides fortes, importância da avaliação da dor e da boa comunicação. Além disso, o folheto tinha espaço para os pacientes fazerem anotações, sendo ofertada referência de leituras adicional. Para essa intervenção, cada participante foi entrevistado três vezes durante um mês. Essas informações reforçavam o que continha no DVD, além de serem fornecidas para os pacientes e cuidadores levarem para suas residências. O estudo mostrou melhorias no que diz respeito a conhecimento e atitudes, e pequenas melhorias sobre intensidade da dor.

Syrjala et al. (2008) testaram um método de treinamento através de vídeo e manual com conteúdos padronizados. O vídeo e manual foram direcionados para pacientes que apresentavam dor relacionada ao câncer, com condições para aprender e aplicar novas informações. O treinamento, direcionado a enfermeiros, médicos, pacientes e cuidadores, abordou maneiras de ajudar pacientes com câncer a se sentirem mais confortáveis. O conteúdo dos materiais foi determinado por painel de especialistas em oncologia. Os materiais foram projetados para uso diário na prática ambulatorial por enfermeiros e médicos. A linguagem utilizada foi de sexta série, com algumas terminologias específicas além desse nível. 
Quanto ao conteúdo, o manual foi estruturado em quatro seções: 1) O que você quer saber - barreiras comuns ao alívio da dor, conscientização da ampla variedade de opções de tratamento disponíveis e componentes essenciais da comunicação com médicos; 2) efeitos colaterais - sintomas relacionados ao tratamento da dor que podem atrapalhar o tratamento e o que fazer com eles; 3) coisas a dizer ao seu médico - forneceu uma lista de verificação de sintomas para o enfermeiro/médico focar durante uma consulta; e 4) tratamentos - forneceu um gráfico e cartões para cada um dos 26 medicamentos comuns e outros tratamentos de dores. Observou-se que o treinamento da dor para os pacientes com câncer, reduziu significativamente a dor usual e os obstáculos ao alívio da dor. Também houve melhora na comunicação entre pacientes-profissionais de saúde sobre intensidade da dor, bem como um maior uso de opioides (SYRJALA et al., 2008).

Por fim, West et al. (2003) desenvolveram ferramenta intitulada PRO-SELF: Pain Control Program para uso de enfermeiros que prestam assistência a pacientes com câncer. O PRO-SELF (Programa de controle da dor) foi desenvolvido para fornecer aos pacientes e cuidadores informações, desenvolvimento de habilidades e suporte interativo de enfermagem destinado a ensinar os pacientes a prevenir e controlar os efeitos da sua doença e, tratamento. Além disso, visa ensinar e orientar pacientes sobre como adaptar seus analgésicos prescritos e vendidos sem receita para melhor alívio da dor.

Os participantes completaram um questionário sobre seus conhecimentos em relação a dor do câncer, seu manejo, efeitos colaterais dos analgésicos e conceitos de dependência e tolerância e, receberam um livro de controle da dor (PROSELF: Pain Control Book). O PRO-SELF foi escrito com linguagem de oitava série (West et al, 2003). Os enfermeiros realizaram o treinamento com os pacientes para que eles pudessem: desenvolver habilidades em avaliar sua dor e sua resposta aos analgésicos; desenvolver uma gestão analgésica que melhor atendesse as necessidades de tratamento e estilo de vida; planejar estratégias para prevenção e tratamento de efeitos colaterais dos analgésicos; e conversar com os profissionais de saúde que os acompanhavam caso sintam que a receita analgésica não está adequada. Após aplicar o programa de controle da dor, evidenciou-se que a ferramenta é eficaz, podendo ser utilizada para ajudar pacientes com câncer e seus cuidadores a terem mais conhecimento e a desenvolver as habilidades necessárias para gerenciar a dor (West et al, 2003).

Na Figura 3, apresenta-se a síntese dos temas abordados em cada grupo de ações de educação em saúde sobre dor. 
Figura 3. Síntese das estratégias para educação em saúde sobre dor no hospital.

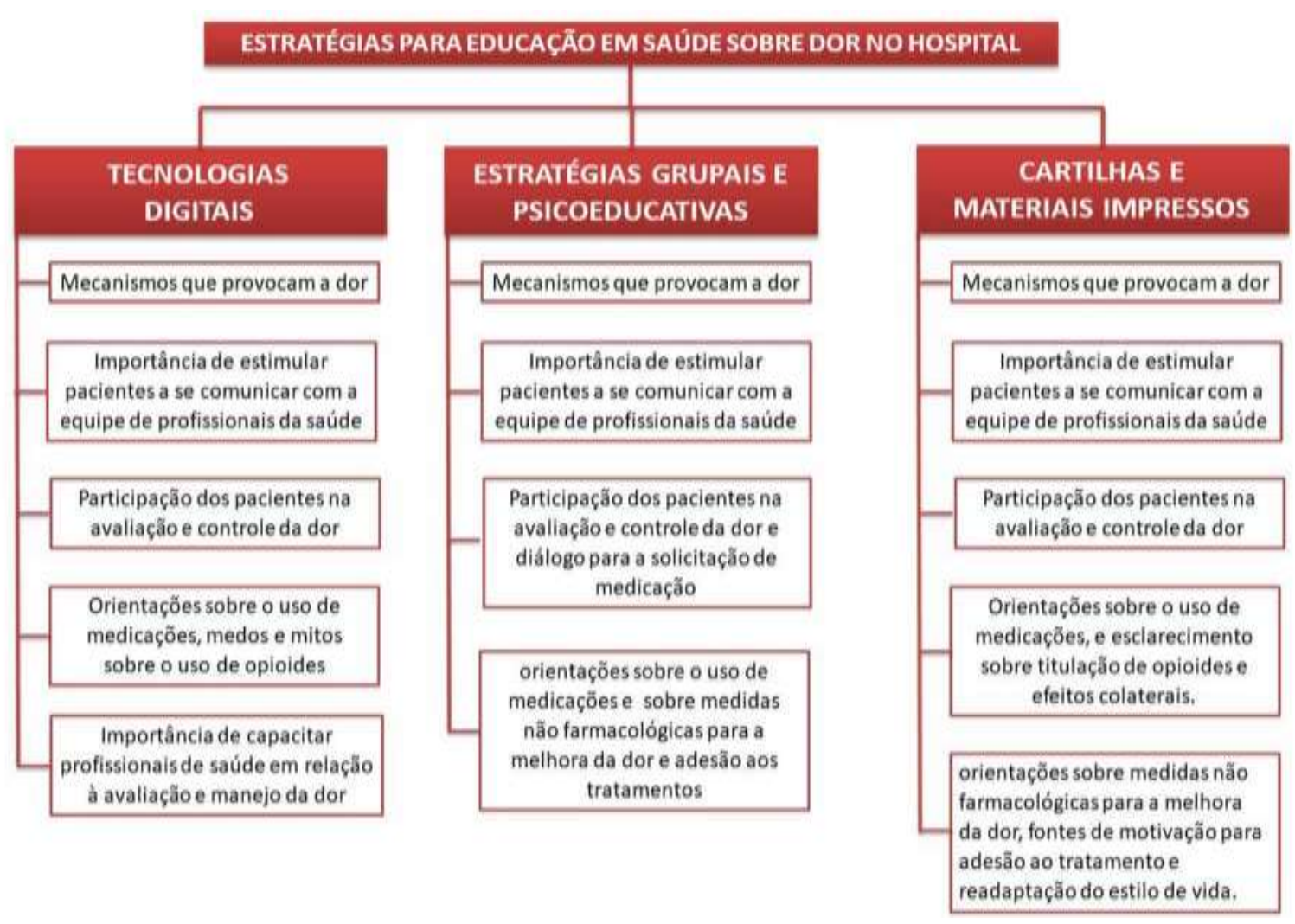

Fonte: Hahn e Cordeiro (2021).

\section{Discussão}

Os estudos analisados nesta revisão permitiram a identificação de estratégias de educação em saúde com bom nível de evidência, tendo a maioria apresentado nível II (Valentim et al., 2019; Capewell, Gregory, Closs \& Bennett, 2010; Street et al., 2010; Syrjala et al., 2008; Marco et al., 2006; Yates et al., 2004), ou seja, são oriundos de ensaios clínicos randomizados bem delineados, e nível III (Chu. et al., 2019; Vieira et al., 2019; Alvarez et al., 2018; Loduca et al., 2018; Sullivan et al., 2018; Edwards et al., 2001; Erkes, Parker, Carr \& Mayo, 2001), que representam evidências derivadas de estudos clínicos não randomizados, mas bem delineados. Além disso, os periódicos nos quais os estudos tiveram maior frequência de publicação possuem fator de impacto acima de 2, o que indica a qualidade e o rigor dos estudos selecionados.

Dito isto, considera-se que os estudos possuem força de recomendação para utilização na prática clínica dos profissionais. Assim, ao abordar a dor, em ações educativas, é recomendado o uso de vídeo associado com folheto ou cartilha, vídeo isolado, orientação verbal associada com folheto ou cartilha, cartilha ou folheto isolados, jogo, psicodrama e tecnologias digitais como sites ou aplicativos.

Ao encontro dos achados desta pesquisa, vídeo, como estratégia para melhorar o controle da dor se mostrou positivo na pesquisa de Selhorst, Hoehn, Degenhart, Schmitt e Fernandez-Fernandez (2020). Os autores desenvolveram, com o auxílio de terapeuta em psicanálise, um vídeo com intervenções psicológicas direcionadas as adolescentes com dor patelo femoral. O vídeo abordava cinco dimensões cognitivas envolvendo a doença de base e a dor: esforço para avaliar o sintoma e explicar a doença, crença sobre o que provoca a resposta à dor; expectativa de quanto tempo à sensação de dor dura; expectativa de como a doença afeta a vida e diferentes áreas de sua vida e capacidade pessoal de mudança. Concluiu-se que o uso do vídeo com informações psicológicas associadas ao atendimento do fisioterapeuta pode reduzir as crenças desadaptativas em adolescentes com do patelo femoral, sendo potencialmente benéfico àqueles com dor crônica. 
Outro estudo (Dalmolin, Girardon-Perlini, Coppetti, Rossato, Gomes \& Silva, 2016), que teve por objetivo conhecer as percepções de participantes de um grupo de apoio para pessoas com colostomia sobre a utilização de um vídeo educativo, corrobora a potência da utilização de vídeo como ação de educação em saúde. Nele, 16 pacientes assistiram a um vídeo de oito minutos e 53 segundos que simulava situações reais de pacientes em uso de colostomia por câncer. Ao final, percebeu-se que o vídeo contribuiu para o processo de aceitação, adaptação do cuidado diante da necessidade de uso de colostomia. Sendo considerada assim, uma ferramenta a ser incorporada no preparo dos pacientes e da família para o autocuidado no domicílio.

Para além da educação de pacientes e familiares, o uso do vídeo também pode ser considerado na educação de estudantes e profissionais de saúde. Ratificando os resultados encontrados, Gagnon, Hadjistavropoulos e Williams (2013) desenvolveram e avaliaram, no Canadá, um vídeo de treinamento sobre avaliação da dor em adultos mais velhos e o impacto do vídeo em profissionais de saúde de clínicas de cuidados prolongados. O vídeo, de 45 minutos, abordava as vantagens do uso da avaliação da dor nessas instituições, medidas de autorrelato para avaliação da dor para indivíduos com demência leve a moderada e abordagens clínicas padronizadas. Como resultado, os participantes, especialmente os enfermeiros, sentiram-se satisfeitos com o formato do vídeo, o conteúdo e as informações. Auxiliares de cuidado apresentaram mais dificuldade de compreensão, entretanto, a ferramenta demonstrou-se efetiva na incorporação e aprimoramento dos conhecimentos das equipes e intervenção na prática sobre a dor de idosos hospitalizados em clínicas de cuidados prolongados.

Quanto ao uso de cartilhas, elas têm se demonstrado eficientes na abordagem e controle da dor. Estudo australiano, no qual foi desenvolvida e validada uma cartilha sobre dor lombar e os aspectos que a envolvem, com base na ferramenta "Roda da Mudança de Comportamento" (Behaviour Change Wheel - $B C W$ ), constatou redução da sensação de dor e medo pelos pacientes, além do uso moderado de exames de imagens e maior aderência às recomendações de diretrizes clínicas na tomada de decisão em relação ao tratamento por parte dos médicos (Simula et al, 2019).

Na mesma direção, entretanto com população de estudo diferente, pesquisa chinesa (Yu, Chair, Chan \& Choi, 2015), que avaliou a eficácia de um livreto de educação em saúde e acompanhamentos por telefone na adesão aos medicamentos, qualidade de vida relacionada à saúde e ao estado psicológico de pacientes com insuficiência cardíaca congestiva, constatou que tal abordagem pode ser útil e incorporada no planejamento e preparo da alta hospitalar e no domiciliar. Com um baixo custo das intervenções (folheto mais ligações telefônicas), o que as tornam utilizáveis em contextos com menos recursos, os autores perceberam que os paciente apresentaram maior controle da doença, bem-estar físico, psicológico e social.

O psicodrama, outra estratégia evidenciada na presente revisão, é uma forma de abordagem em grupo para solucionar conflitos existenciais ou em relações. Ele recorre ao lúdico e à representação por meio de elementos teatrais acionados em cenas baseadas em situações reais ou imaginárias. Assim, ao utilizar-se da técnica com 15 pessoas com doença oncológica, Antoniassi Junior, Gomes, Beretta e Figueiredo (2018), perceberam que ela possibilitou aos participantes trabalharem as dores emocionais, explorar anseios e desenvolver a sensibilidade diante da experiência do adoecimento pelo câncer.

Também, jogos representam uma estratégia lúdica que pode auxiliar no desenvolvimento de ações de educação em saúde, conforme identificado neste estudo. Ao encontro, pesquisa na qual foi desenvolvido um jogo de tabuleiro para a promoção da saúde cardiovascular de adolescentes, evidenciou essa estratégia como forma de melhorar a participação dos adolescentes no autocuidado, modificando comportamentos e hábitos de vida, de modo a torná-los mais saudáveis (Lima et al., 2020). Outra experiência com jogo foi identificada no estudo de D’ávila, Puggina e Fernandes (2018), em que as autoras desenvolveram um jogo educativo com cartas para orientar gestantes sobre os direitos e as boas práticas relacionadas ao parto. O jogo foi avaliado como compreensível e indicado como estratégia auxiliar na orientação das mulheres durante a gestação.

Em relação ao uso de tecnologias de informação e comunicação, como sites e aplicativos, que foram evidenciados no presente estudo, ele pode ser reforçado pelos resultados da pesquisa de Muñoz-Narbona, Cabrera-Jaime, Lluch-Canut, Castaño e Roldán-Merino (2020). Esses autores realizam uma intervenção por meio de curso em plataforma online sobre dor com 
enfermeiros de dois hospitais espanhóis. O curso fazia parte de um estudo de validação de uma escala modificada para avaliação da dor de pacientes com distúrbios neurológicos e câncer não comunicativos, e abordava conceitos gerais sobre dor e avaliação dessa por meio da ferramenta recomendada. Concluiu-se que a intervenção teve boa aceitação e foi eficaz na educação e preparo dos enfermeiros.

\section{Considerações Finais}

Nesta revisão, identificou-se estratégias para o desenvolvimento de educação em saúde sobre dor no hospital. Tais estratégias, que apresentaram, em sua maioria, recomendação forte, podem ser utilizadas nas abordagens com pacientes, cuidadores e também com estudantes e profissionais de saúde.

As limitações do estudo estiveram relacionadas aos idiomas considerados, já que experiências e estratégias podem ter sido perdidas ao restringi-los. Também, se outras bases de dados fossem consultadas, outras pesquisas poderiam ter sido recuperadas. $\mathrm{O}$ fato de não ter sido realizada dupla coleta também pode ser um limitador dos resultados encontrados.

De qualquer maneira, como contribuições para a prática assistencial, destaca-se que ao invés de métodos impositivos e expositivos de educação em saúde, vídeos, jogos, cartilhas, psicodrama e plataformas online parecem ser mais atraentes, além de serem mais acessíveis ao tempo e desejo do usuário. Assim, essas estratégias podem ser incorporadas durante o processo de hospitalização, a fim de favorecer a compreensão e o protagonismo de pacientes e familiares no controle da dor. De igual modo, podem ser utilizadas no plano de alta, estimulando a adesão às medidas farmacológicas e não-farmacológicas para o controle do sintoma, com continuidade no domicílio.

Em se tratando de contribuições para o ensino, as mesmas ferramentas também podem facilitar o processo de aprendizagem de estudantes de graduação na área da saúde, ao mesmo tempo em que podem tornar mais dinâmicas as propostas de educação permanente e educação em serviço dos profissionais que atuam em unidades clínicas nos hospitais, os quais precisam de capacitações constantes para avaliar e tratar com as melhores evidências os pacientes com dor.

Por fim, sugere-se que outras pesquisas sejam realizadas junto a pacientes, cuidadores, profissionais e estudantes de saúde, de modo a verificar as repercussões e implicações práticas, em contextos específicos, do uso das estratégias identificadas para a melhor abordagem da dor.

\section{Referências}

Alves Neto, O. et al. (2009). Dor: princípios e práticas. Artmed.

Alvarez, A. et al. (2018). Análise de qualidade de objeto virtual de aprendizagem para avaliação da dor em enfermagem. Revista Cubana de Enfermería, 34(3). http://www.revenfermeria.sld.cu/index.php/enf/article/view/1447/375

Antoniassi Junior, G., Gomes, G. A., Beretta, R. C. S., \& Figueiredo, G. L. A. (2020). Psychodrama in the promotion of health and well-being: group experience with cancer patients. Revista Brasileira De Psicodrama, 26(2), 133-139. https://revbraspsicodrama.org.br/rbp/article/view/132

Brasil. (2012). Protocolo Clínico e Diretrizes Terapêuticas. Portaria SAS/MS $n^{o} 1083$, 02 de outubro de 2012. Brasília: Ministério da Saúde. http://portalarquivos.saude.gov.br/images/pdf/2016/novembro/23/Dor-Cronica---PCDT-Formatado---com-escala-de-dor-LANSS.pdf.

Buchbinder, M., Wilbur, R., McLean, S., \& Sleath, B. (2015). "Is there any way I can get something for my pain?" Patient strategies for requesting analgesics. Patient education and counseling, 98(2), 137-143. https://doi.org/10.1016/j.pec.2014.10.021

Capewell, C., Gregory, W., Closs, S., \& Bennett, M. (2010). Brief DVD-based educational intervention for patients with cancer pain: feasibility study. Palliative medicine, 24(6), 616-622.: https://doi.org/10.1177/0269216310371704

Chu, T. L., et al. (2019). Multimedia-assisted instruction on pain assessment learning of new nurses: a quasi-experimental study. BMC Med Educ,19(68). https://doi.org/10.1186/s12909-019-1496-z

Dalmolin, A., Girardon-Perlini, N. M. O., Coppetti, L. C., Rossato, G. C., Gomes, J. S., \& Silva, M. E. N. (2016). Vídeo educativo como recurso para educação em saúde a pessoas com colostomia e familiares. Revista Gaúcha de Enfermagem, 37, e68373. https://doi.org/10.1590/1983-1447.2016.esp.68373 
D'Avila, C. G., Puggina, A. C., \& Fernandes, R. A. Q. (2018). Construção e validação de jogo educativo para gestantes.Escola Anna Nery, $22(3)$, e20170300. https://doi.org/10.1590/2177-9465-ean-2017-0300

Dik, A. B., \& Lohmann, P. M. (2020). A dor no contexto da urgência e emergência: uma revisão integrativa. Research, Society and Development, 9(4), e105942898. https://doi.org/10.33448/rsd-v9i4.2898

Edwards, H. E. et al. (2001). Improving pain management by nurses: A pilot Peer Intervention Program. Nursing \& health sciences, 3(1), 35-45. https://doi.org/10.1046/j.1442-2018.2001.00069.x

Erkes, E. B., Parker, V. G., Carr, R. L., \& Mayo, R. M. (2001). An examination of critical care nurses' knowledge and attitudes regarding pain management in hospitalized patients. Pain management nursing: official journal of the American Society of Pain Management Nurses, 2(2), 47-53. https://doi.org/10.1053/jpmn.2001.23177

Ferrari, M. F. M., Daher, D. V., Antunes, J. M., Amim, E. F., Jesus, C. M., \& Geraldo, M. A.(2019). A dor como quinto sinal vital, desafios para a incorporação na formação em saúde. REME - Rev Min Enferm., 23, e-1233. 10.5935/1415-2762.20190081

Gagnon, M. M., Hadjistavropoulos, T., \& Williams, J. (2013). Development and mixed-methods evaluation of a pain assessment video training program for long-term care staff. Pain Res Manag, 18(6), 307-312. https://doi.org/10.1155/2013/659320

International Association for the study of pain (IAS). (2017). Terminology. https://www.iasppain.org/Education/Content.aspx?ItemNumber=1698\&navItemNumber=576\#Pain.

Knaul, F. M. et al. (2018). Alleviating the access abyss in palliative care and pain relief-an imperative of universal health coverage: the Lancet Commission report. Lancet, 391(10128), 1391-1454. https://doi.org/10.1016/S0140-6736(17)32513-8

Kuehn B. (2018). Chronic Pain Prevalence. JAMA,320(16), 1632. doi:10.1001/jama.2018.16009

Lima, N. K. G. et al. (2020). Proposta de jogo como tecnologia educacional para a promoção da saúde cardiovascular do adolescente. Braz. J. Hea. Rev.,3(5), p. 13494-13514. 10.34119/bjhrv3n5-173

Lima, L. \& Reis, F. (2018). The use of an E-pain technology in the management of chronic pain. Case report. BrJP, 1(2), 184-187. http://dx.doi.org/10.5935/2595-0118.20180036

Loduca, A., Müller, B. M., Focosi, A. S., Samuelian, C., \& Yeng, L. T. (2018). How interdisciplinary psychoeducational programs with a psychodrama approach can help the chronic pain treatment compliance. BrJP, 1(2), 122-126. http://dx.doi.org/10.5935/2595-0118.20180024

Marco, C. A. et al. (2006). The verbal numeric pain scale: effects of patient education on self-reports of pain. Academic emergency medicine: official journal of the Society for Academic Emergency Medicine, 13(8), 853-859. https://doi.org/10.1197/j.aem.2006.04.020

Melnyk, B. M., \& Fineout-Overholt, E. (2019). Making the case for evidence-based practice. In Melnyk B. M, Fineout-Overholt E (Ed.), Evidence-based practice in nursing \& healthcare: a guide to best practice (pp. 35-62). Philadelphia: Lippincot Williams \& Wilkins.

Mendes, K. S., Silveira, R. C. C. P., \& Galvão, C. M. (2019). Uso de gerenciador de referências bibliográficas na seleção dos estudos primários em revisão integrativa. Texto \& Contexto - Enfermagem, 28, e20170204. https://doi.org/10.1590/1980-265x-tce-2017-0204

Mendez, S. P., Sá, K. N., Araújo, P. C. S., Oliveira, I. A. V. F., Gosling, Artur P., \& Baptista, A. F. (2017). Elaboration of a booklet for individuals with chronic pain. Revista Dor, 18(3), 199-211.https://doi.org/10.5935/1806-0013.20170103

Miranda, C. C. V., Seda Junior, L. F., \& Pelloso, L. R. C. A. (2016). Nova classificação fisiológica das dores: o atual conceito de dor neuropática. Revista Dor, 17(Suppl. 1), 2-4. https://doi.org/10.5935/1806-0013.20160037

Moisset, X. et al. (2020). Pharmacological and non-pharmacological treatments for neuropathic pain: Systematic review and French recommendations. Revue Neurologique, 176(5), p. 325-352. 10.1016/j.neurol.2020.01.361

Muñoz-Narbona, L., Cabrera-Jaime, S., Lluch-Canut, T., Castaño, P. B., \& Roldán-Merino, J. (2020). E-Learning course for nurses on pain assessment in patients unable to self-report. Nurse Education in Practice, 43, 102728. doi:10.1016/j.nepr.2020.102728

North American Nursing Diagnosis Association International (NANDA-I). (2018). Diagnósticos de enfermagem da NANDA: definições e classificação 20182020. 11 a ed. Porto Alegre: Artmed.

Pedroso, J. K. N. et al. (2017). Dor em oncologia: percepção do paciente e dos profissionais de enfermagem. Revista Cubana de Enfermería, 33(4), e1020. Disponível em: http://www.revenfermeria.sld.cu/index.php/enf/article/view/1020/309

Selhorst, M., Hoehn, J., Degenhart, T., Schmitt, L., \& Fernandez-Fernandez, A. (2020).Psychologically-informed video reduces maladaptive beliefs in adolescents with patellofemoral pain. Physical Therapy in Sport, 41, 23-28. doi:10.1016/j.ptsp.2019.10.009

Simula et al. B. (2019). Transcultural adaption and preliminary evaluation of "understanding low back pain" patient education booklet. BMC Health Services Research, 19, 1010. https://doi.org/10.1186/s12913-019-4854-y

Santana, J. M., Perissinotti, D. M. N., Oliveira Junior, J. O., Correia, L. M. F., Oliveira, C. M., \& Fonseca, P. R. B. (2020). Definição de dor revisada após quatro décadas. BrJP, 3(3), 197-198. https://doi.org/10.5935/2595-0118.20200191

Sullivan, M. et al. (2018). A Controlled Pilot Trial of PainTracker Self-Manager, a Web-Based Platform Combined With Patient Coaching, to Support Patients' Self-Management of Chronic Pain. The journal of pain, 19(9), 996-1005. https://doi.org/10.1016/j.jpain.2018.03.009 
Research, Society and Development, v. 10, n. 3, e25210313297, 2021

(CC BY 4.0) | ISSN 2525-3409 | DOI: http://dx.doi.org/10.33448/rsd-v10i3.13297

Street, R. L. et al. (2010). Improving physician-patient communication about cancer pain with a tailored education-coaching intervention. Patient education and counseling, 80(1), 42-47. https://doi.org/10.1016/j.pec.2009.10.009

Syrjala, K. L. et al. (2008). Patient training in cancer pain management using integrated print and video materials: a multisite randomized controlled trial. Pain, 135(1-2), 175-186. https://doi.org/10.1016/j.pain.2007.10.026

Valentim, J. C. Paiva, Meziat-Filho, N. A., Nogueira, L. C., \& Reis, F. J. J. (2019). ConheceDOR: the development of a board game for modern pain education for patients with musculoskeletal pain. $B r J P, 2(2), 166-175$. https://doi.org/10.5935/2595-0118.20190030

Vieira, A. S. M., Castro, K. V., Canatti, J. R., Oliveira, I. A. V. F., Benevides, S. D. \& Sá, K. N. (2019). Validation of an educational booklet for people with chronic pain: EducaDor. BrJP, 2(1), 39-43. https://doi.org/10.5935/2595-0118.20190008 